\title{
JUURNAL.RU
}

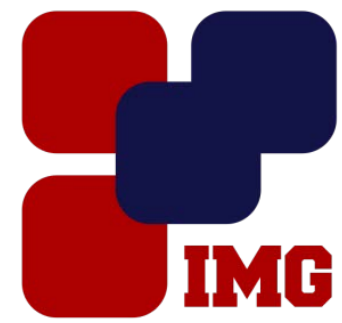
IYANOY Management GROUP

\author{
Буткай Д.В., Струков П. С., Суконщиков А.А., Бахтенко Е.А. \\ Вологодский государственный университет \\ Вологда, Россия
}

doi: 10.18411/lj-31-01-2017-1-03

idsp 000001:lj-31-01-2017-1-03

\section{Математический аппарат для описания и графического представления контекстно-свободных грамматик искусственных языков: синтаксис формул без переменных}

Рассмотрим определение искусственного языка и его математической модели по аналогии с определением формального языка и его математической модели. Формальный язык определяется как множество текстов, которые могут быть написаны на этом языке. Искусственный язык определим как множество смыслов, которые могут быть выражены на этом языке.

Математической моделью текста является конечная строка, состоящая из знаков, принадлежащих заданному конечному набору. Таким образом, математической моделью (экстенсионалом) формального языка является некоторое (бесконечное) множество таких строк. Будем считать, как это принято в области искусственного интеллекта, математической моделью смысла (некоторой информации) конечную семантическую сеть, т.е. ориентированный размеченный связный граф без циклов, содержащий конечное число вершин. Способы представления смыслов (информации) семантическими сетями определяются правилами, специфическими для каждого искусственного языка; их рассмотрение выходит за рамки данной работы. Таким образом, математической моделью (экстенсионалом) искусственного языка будем считать некоторое (бесконечное) множество конечных семантических сетей.

Грамматикой, порождающей некоторый формальный язык, является исчисление, порождающее экстенсионал этого формального языка. Грамматикой, порождающей некоторый искусственный язык, будем считать исчисление, порождающее экстенсионал этого искусственного языка. Грамматика искусственного языка может быть дополнена набором правил (проекцией), позволяющим перейти от смысла (информации) к тексту (выражающему эту информацию), т.е. позволяющим по семантической сети построить соответствующую ей конечную строку из заданного конечного набора знаков. 
Для представления грамматик искусственных языков требуется некоторый метаязык. Поскольку такие грамматики должны определять (бесконечные) подмножества множества семантических сетей, в качестве метаязыка естественно взять логический язык. В этом случае грамматика искусственного языка есть логическая формула этого метаязыка, истинная для тех и только тех семантических сетей, которые принадлежат этому искусственному языку. Поскольку логическая семантика метаязыка (способ вычисления значения истинности его формул) будет определена относительно семантических сетей, т.е. объектов, имеющих структуру, естественно ввести структуру и в синтаксис метаязыка - формулами метаязыка будут определенным образом размеченные графы (с циклами), в каждом из которых выделена одна вершина, называемая начальной вершиной этой формулы.

Наряду с логической семантикой метаязыка будет определена и его порождающая семантика - начальным состоянием порождающего процесса является начальная вершина (корень) порождаемой сети, которая объявляется активной; с каждой вершиной порождаемой сети связывается формула метаязыка; на очередном шаге порождающего процесса из множества активных вершин выбирается одна, из которой выполняется очередной шаг порождения в соответствие с формулой, связанной с этой вершиной; при этом вершина, из которой выполнен шаг порождения, перестает быть активной, но могут возникнуть новые активные вершины; порождающий процесс заканчивается, когда в порождаемой сети нет ни одной активной вершины. Логическая и порождающая семантики метаязыка связаны следующим естественным условием - по каждой формуле метаязыка может быть порождено множество тех и только тех семантических сетей, относительно которых эта формула истинна.

В свою очередь иерархическая семантическая сеть есть связный ориентированный граф без циклов, в котором каждая дуга имеет метку, а вершины могут быть одного из двух типов - простые и структурные; одна из вершин сети, в которую не входит ни одна дуга, выделяется и называется ее корнем. Каждая простая терминальная вершина сети имеет в качестве метки константу некоторого сорта. Корень сети имеет две метки-термина, из которых первая метка есть метка искусственного языка (класса), а вторая индивидуальная метка сети. Каждая структурная вершина сети является контейнером, содержащим упорядоченное конечное множество иерархических семантических сетей (далее по тексту — «сеть») с одной и той же меткой класса и попарно различными индивидуальными метками.

Контекстно-свободные грамматики искусственных языков.Контекстносвободная грамматика искусственного языка есть формула без переменных метаязыка, начальная вершина которой имеет метку - название этого искусственного языка.

Синтаксис формул без переменных. Формула без переменных метаязыка может относиться к одной из следующих групп: 
- простая формула без переменных;

- простая кванторная формула без переменных;

- унарная формула без переменных;

- пропозициональная формула без переменных;

- структурная кванторная формула без переменных.

Простая формула без переменных есть граф, состоящий из единственной вершины с меткой с (рис. 1). Эту единственную вершину будем называть начальной вершиной простой формулы без переменных. Меткас является константой.

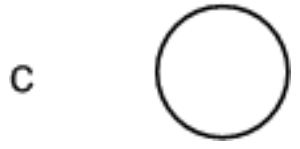

Рис. 1. - Простая формула без переменных

Простая кванторная формула без переменных есть граф, состоящий из единственной вершины с меткой, имеющей вид QMT, где Q - знак квантора, M описание (конечного или бесконечного) множества, а Т- термин (рис. 2). Знаком квантора может быть: $\forall$ (для всех), $\exists$ (существует), $\exists 2$ (существует не менее двух), $\exists$ ? (существует, но не для всех), $\exists$ ! (существует и единственен), $\exists[]$ (существует подъинтервал). Множество кванторов является расширяемым.

\section{QMT}

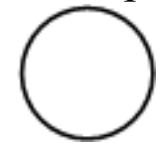

Рис. 2. - Простая кванторная формула без переменных

Описание конечного множества может иметь вид $\{\mathrm{c} 1, \ldots, \mathrm{cn}\}$, где $\mathrm{c} 1, \ldots$, cn - попарно различные константы, либо быть целым конечным интервалом; в этом случае знаком квантора может быть $\forall, \exists, \exists 2, \exists$ ? или $\exists$ !. Описание бесконечного множества может быть названием сорта, неименованным множеством «*», или вещественным конечным интервалом; в этом случае знаком квантора может быть $\exists, \exists 2, \exists$ !. Если знак квантора есть $\exists[]$, то описанием множества может быть только конечный целый или вещественный интервал.

Названием сорта может быть: "строка", "целый", "вещественный", "целый интервал", "вещественный интервал", "дата-время". Множество сортов является расширяемым.

Унарная формула без переменных есть граф, состоящий из начальной вершины и дуги с меткой Т (термином), выходящей из начальной вершины и входящей в начальную вершину некоторой формулы без переменных F (рис. 3).

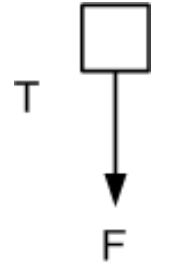

Рис. 3. - Унарная формула без переменных 
Пропозициональная формула без переменных есть граф, состоящий из начальной вершины с пропозициональной меткой Р и выходящих из нее $\mathrm{n}$ дуг (не менее двух), каждая из которых имеет метку Ті (термин; і от 1 до n; все эти метки должны быть попарно различны) и входит в начальную вершину некоторой формулы без переменных Fi (рис. 4).

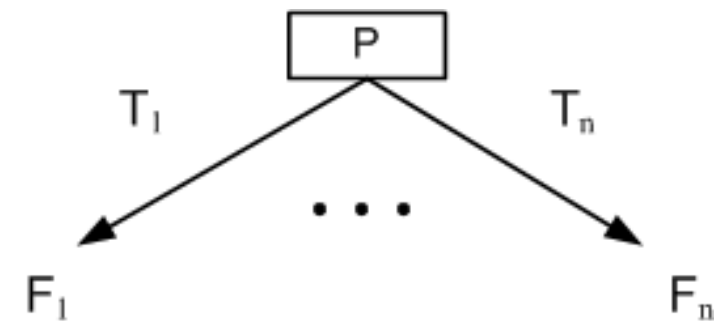

Рис. 4. - Пропозиииональная формула без переменныхх

Пропозициональными метками Р являются:

\& - конъюнкция;

$\checkmark$ - дизъюнкция;

| - исключающее или.

Множество пропозициональных меток является расширяемым.

Любая из дуг, выходящая из начальной вершины с пропозициональной меткой \&, может иметь метку факультативности "[]".

Структурная кванторная формула без переменных состоит из начальной вершины с меткой, имеющей вид QMT, где Q - знак квантора, M - описание (конечного или бесконечного) множества, а $\mathrm{T}$ - термин, и формулы без переменных F, начальная вершина которой изображается внутри начальной вершины структурной кванторной формулы без переменных (рис. 5).

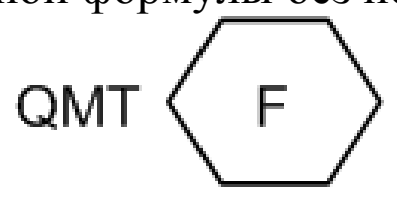

Рис. 5. - Структурная кванторная формула без переменных

Описание конечного множества может иметь вид $\{\mathrm{c} 1, \ldots, \mathrm{cn}\}$, где $\mathrm{c} 1, \ldots$, cn - попарно различные константы, либо быть целым конечным интервалом; в этом случае знаком квантора может быть $\forall, \exists, \exists 2, \exists$ ?. Описание бесконечного множества может быть названием сорта, неименованным множеством «*», или вещественным конечным интервалом; в этом случае знаком квантора может быть $\exists, \exists 2$. 